\title{
Repeated state change of variable gamma-ray pulsar, PSR J2021+4026
}

\author{
J. Takata ${ }^{1}$, H.H.Wang ${ }^{1}$, L.C.C. Lin $^{2}$, C.-P. Hu ${ }^{3.4}$, C.Y. Hui ${ }^{5}$, A.K.H. Kong ${ }^{6}$, P.H.T. Tam ${ }^{7}$, \\ K.L. Li ${ }^{2,6}$, K.S. Cheng ${ }^{8}$ \\ takata@hust.edu.cn
}

\begin{abstract}
PSR J2021+4026 is a radio-quiet gamma-ray pulsar and the first pulsar that shows state change of the gamma-ray emission and spin-down rate. The state change of PSR J2021+4026 was first observed at 2011 October, at which the pulsar changes the state from high gamma-ray flux/low spin-down rate state to low gamma-ray flux/high spin-down rate state. In December 2014, PSR J2021+4026 recovered the state before the 2011 state change over a timescale of a few months. We report that the long term evolution of the gamma-ray flux and timing behavior suggests that PSR J2021+4026 changed the state near 2018 February 1st and entered a new low gamma-ray flux/high spin-down rate state. At the 2018 state change, the averaged flux dropped from $(1.29 \pm 0.01) \times 10^{-6} \mathrm{cts} \mathrm{cm}^{-2} \mathrm{~s}^{-1}$ to $(1.12 \pm 0.01) \times 10^{-6} \mathrm{cts}_{\mathrm{cm}}^{-2} \mathrm{~s}^{-1}$, which is behavior similar to the case of the 2011 event. The spin-down rate has increased by $\sim 3 \%$ in the new state since the 2018 state change. The shapes of pulse profile and spectrum in $\mathrm{GeV}$ bands also changed at the 2018 event, and they are consistent with behavior at the 2011 state change. Our results probably suggest that PSR J2021+4026 is switching between different states with a timescale of several years, like some radio pulsars
\end{abstract}

\footnotetext{
${ }^{1}$ School of physics, Huazhong University of Science and Technology, Wuhan, 430074, China

${ }^{2}$ Department of Physics, UNIST, Ulsan 44919, Korea

${ }^{3}$ JSPS International Research Fellow

${ }^{4}$ Department of Astronomy, Kyoto University, Oiwakecho, Sakyoku, Kyoto 606-8502, Japan

${ }^{5}$ Department of Astronomy and Space Science, Chungnam National University, Daejeon 305-764, Republic of Korea

${ }^{6}$ Institute of Astronomy and Department of Physics, National Tsing Hua University, Hsinchu, Taiwan

${ }^{7}$ School of Physics and Astronomy, Sun Yat-Sen University, Zhuhai, 519082, China

${ }^{8}$ Department of physics, The University of Hong Kong, Pokfulam Road, Hong Kong
} 
(e.g. PSR B1828-11). PSR J2021+4026 will provide a unique opportunity to study the mechanism of the state switching.

\section{Subject headings:}

\section{Introduction}

PSR J2021+4026 (3FGL J2021.5+4026) is one of the brightest radio-quiet gamma-ray pulsars (Abdo et al. 2009) with a spin period of $P_{s} \sim 0.265 \mathrm{~s}$ and a spin-down power of $L_{s d} \sim 3.4 \times 10^{36} \mathrm{erg} \mathrm{s}^{-1}$. This pulsar is also known as the first variable gamma-ray pulsar (Allafort et al. 2013, hereafter Al13). Fermi-Large Area Telescope (Fermi-LAT) observed a sudden gamma-ray flux drop near MJD 55,850 (2011 October) and a change of the observed flux $(>100 \mathrm{MeV})$ from $(8.33 \pm 0.08) \times 10^{-10} \mathrm{erg} \mathrm{cm}^{-2} \mathrm{~s}^{-1}$ to $(6.83 \pm 0.13) \times 10^{-10} \mathrm{erg} \mathrm{cm}^{-2} \mathrm{~s}^{-1}$ over a timescale of less than a week. The timing analysis also revealed that the spin-down rate changed from $-(7.8 \pm 0.1) \times 10^{-13} \mathrm{~Hz} \mathrm{~s}^{-1}$ to $-(8.1 \pm 0.1) \times 10^{-13} \mathrm{~Hz} \mathrm{~s}^{-1}$. Subsequent studies (Ng et al. 2016; Zhao et al. 2017) found that the gamma-ray flux and the spin-down rate did not show a gradual recovery toward the values before 2011 October, and the pulsar stayed at low gamma-ray flux and high spin-down rate state (hereafter LGF/HSD state) for about three years after the state change. These flux and timing behaviors suggest that the state change of PSR J2021+4026 is owing to the change of the structure of the global magnetosphere. Around 2014 December, PSR J2021+4026 returned to the high gamma-ray flux/low spin-down rate state (hereafter HGF/LSD state) over a timescale of a few months.

PSR J2021+4026 stayed at the HGF/LSD state for about three years since 2014 December. The aperture photometry light curve (time resolution of 30 days) of PSR J2021+4026 provided by the Fermi science team 11 clearly shows that a gamma-ray flux change occurred around MJD 58,150 (2018 February 1st) and that PSR J2021+4026 entered new low gammaray flux state. Considering that the previous flux change accompanied with a change in the spin-down rate, it is likely that there was another state change in the spin-down rate near MJD 58,150. In the previous study (Zhao et al. 2017), we investigated the evolution of the gamma-ray flux and spin-down rate until MJD 57,700 (2016 November 8th). In this study, therefore, we perform timing and spectral analyses for gamma-ray emission of PSR J2021+4026 with the data after MJD 57,700 in sections 2 and 3 . We will discuss the possible mechanism of the state switching of the PSR J2021+4026 in section 4.

\footnotetext{
${ }^{1}$ https://fermi.gsfc.nasa.gov/ssc/data/access/lat/4yr_catalog/ap_lcs/lightcurve_3FGLJ2021.5p4026.png
} 


\section{Fermi data reduction}

We use the Fermi-LAT Pass 8 (P8R2) data in the energy range of $0.1-300 \mathrm{GeV}$ for the data analysis. To obtain the temporal evolution of the flux and spin-down rate, we select the data from 2016 November 8 to 2019 March 1 in a $20^{\circ}$ radius region of interest (ROI) centered at the 3FGL J2021.5+4026 position. The light curve of PSR J2021+4026 is obtained at $E>0.1 \mathrm{GeV}$ using the binned likelihood analysis in the Fermi Science Tools. We select events in the class for the point source or Galactic diffuse analysis (event class=128) and consider the photons collected in the front and back sections of the tracker (evttype $=3$ ). We exclude events with zenith angles larger than $90^{\circ}$ to reduce the Earth's albedo gamma-ray contamination.

For the spectral analysis, we input spectral models, which are provided by the Fermi Science Support Center2, for the galactic diffuse emission (gll_iem_v06), the isotropic diffuse emission (iso_P8R2_SOURCE_V6_v06) and all the 3FGL catalog sources within $20^{\circ}$ of PSR J2021+4026 to account for the spectral contribution. We also include the extended sources, i.e. the Cygnus-X cocoon and Cygnus Loop, near the PSR J2021+4026. We first fit our data over the entire time range and obtain spectral parameters for the input sources. In this study, the spectrum of PSR J2021+4026 is modeled with a power law plus exponential cut-off function of the form

$$
d N / d E \propto E^{-\Gamma} \exp \left[-\left(E / E_{c}\right)\right],
$$

where $E$ is the energy of the photon, $\Gamma$ is the photon index and $E_{c}$ is the cut-off energy. To obtain the best-fitting spectral parameters, we perform a binned likelihood analysis using the Fermi Science Tools gtlike. We divide the entire energy range (0.1-300GeV) into 12 energy bins with a logarithmic scale, and best-fit spectral parameters are obtained with the elimination of insignificant sources $(<3 \sigma)$. The $1 \sigma$ uncertainty of the fitting parameters is provided by gtlike tool, and it is calculated by the method discussed in (Mattox et al. 1996).

We divide the entire time range (from 2016 November 8 to 2019 March 1) into 30 day time bins and obtain the long temporal evolution of the flux in $E>0.1 \mathrm{GeV}$ bands. For each bin, the contribution of each background source is calculated with the spectral parameters that are obtained from the entire data. For PSR J2021+4026, we refit the data by the binned likelihood analysis (gtlike) and estimate the flux.

To obtain ephemeris and the pulse profile of PSR J2021+4026 after 2018 state change, we extract the source events within $1^{\circ}$ radius centered at the target. The time of arrival of all

\footnotetext{
${ }^{2}$ https ://fermi.gsfc.nasa.gov/ssc/
} 
photons are corrected to TDB (barycentric dynamical time) with the task gtbary supported by Fermi Science Tools.

\section{Results}

\subsection{Evolutions of the gamma-ray flux and spin-down rate}

Top panel of Figure 1 shows the long term evolution for the $>0.1 \mathrm{GeV}$ flux with 30 day time bins that was used in Al13. In addition to the previous event near MJD 55,850 reported in Al13, an obvious flux change occurred at around MJD 58,100-58,200. The photon flux dropped by about $13 \pm 1 \%$ from $F=(1.29 \pm 0.01) \times 10^{-6} \mathrm{cts} \mathrm{cm}^{-2} \mathrm{~s}^{-1}$ averaged over

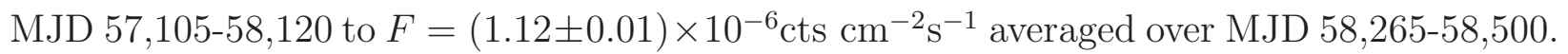
The size of the flux drop for the new event is consistent with $11 \pm 1 \%$ of the previous event within the error (Table 3).

In the previous flux change, a sudden change of the spin-down rate was observed. To examine the evolution of the spin-down rate at around the epoch of the new flux drop, we divide the Fermi-LAT data after $\sim$ MJD 57,700 into a 70 day time bin, which can provide enough photons to detect the pulsation (Zhao et al. 2017). During the analysis, we notice that no Fermi-LAT data for PSR J2021+4026 is available between MJD 58,190 and 58,240, and we exclude this large observational gap when we bin the Fermi data. For each time bin, we describe the frequency evolution as $f(t)=f\left(t_{0}\right)+\dot{f}\left(t_{0}\right)\left(t-t_{0}\right)$, where $f$, $\dot{f}$ and $t_{0}$ represent the spin frequency, the frequency time derivative and reference time, respectively. For reference time $\left(t_{0}\right)$, we choose the middle point of each 70 day time bin. We perform an $H$-test (de Jager \& Büsching 2010) to search the best frequency $f\left(t_{0}\right)$ and the time derivative $\dot{f}\left(t_{0}\right)$ in the range of $[3.767 \mathrm{~Hz}, 3.769 \mathrm{~Hz}]$ and of $\left[-9 \times 10^{-13} \mathrm{~Hz} / \mathrm{s},-7 \times 10^{-13} \mathrm{~Hz} / \mathrm{s}\right]$, respectively, within which the best solutions are expected from the previous analyses (Al13; Zhao et al. 2017). The bottom panel of Figure 1 shows the evolution of $\dot{f}$, and the uncertainty of each data point denotes the Fourier resolution in the periodicity search. In each data set, we can clearly identify the pulse peak in the folded light curve with the local ephemeris.

In Figure 1, we can see a change of the spin-down rate $(\dot{f})$ around the epoch of the gamma-ray flux drop. No recovery of the spin-down rate and gamma-ray flux are observed since $\sim$ MJD 58,150. This indicates that PSR J2021+4026 changed the state from a HGF/LSD state to a LGF/HSD state around MJD 58,150 (2018 February 1st). The averaged spin-down rates at the four stages (dashed lines in the bottom panel of Figure 1), are $\dot{f}=-7.72 \pm 0.05 \times 10^{-13} \mathrm{~Hz} \mathrm{~s}^{-1},-8.15 \pm 0.04 \times 10^{-13} \mathrm{~Hz} \mathrm{~s}^{-1},-7.77 \pm 0.05 \times$ $10^{-13} \mathrm{~Hz} \mathrm{~s}^{-1}$ and $-8.01 \pm 0.07 \times 10^{-13} \mathrm{~Hz} \mathrm{~s}^{-1}$, which are calculated from the data points at 


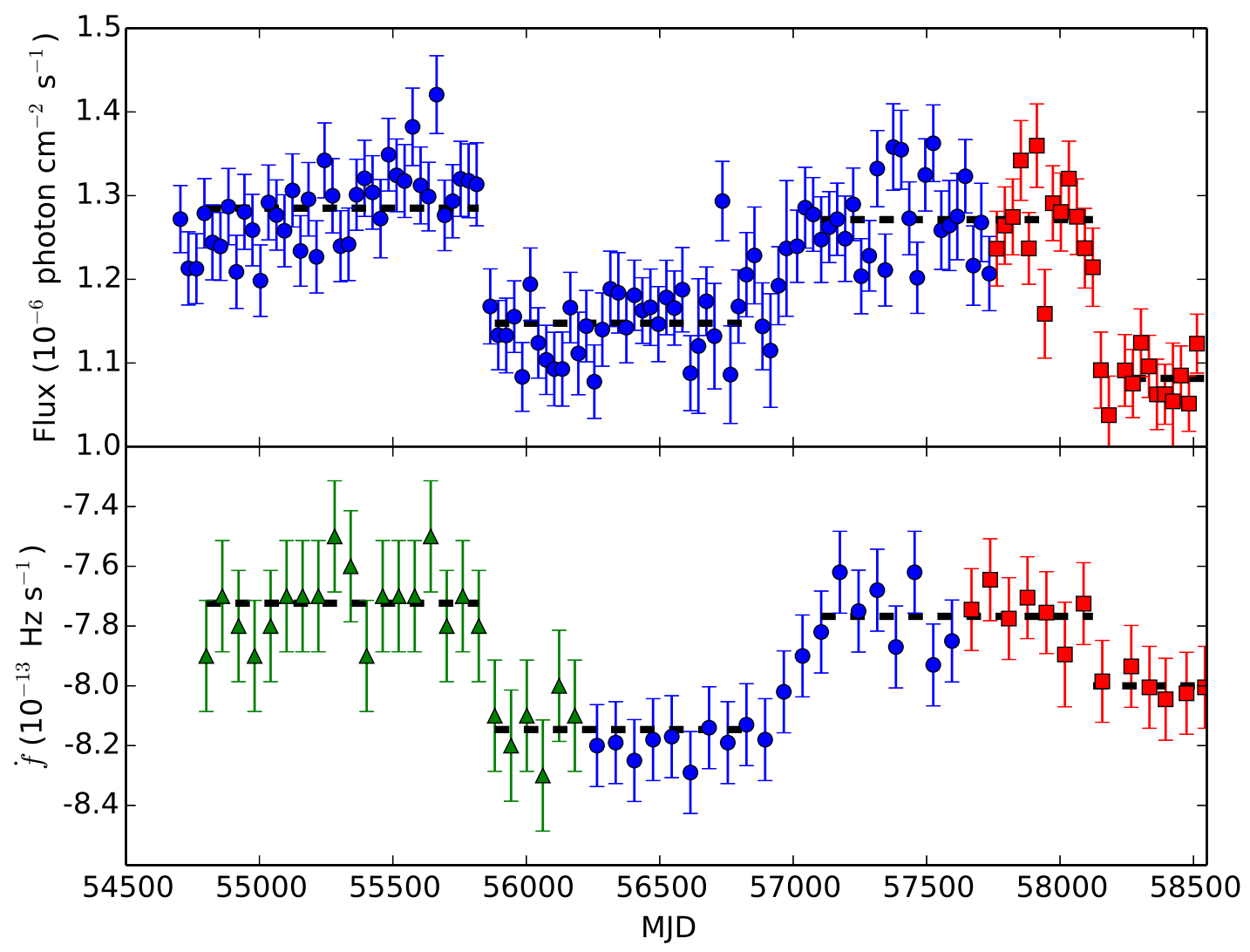

Fig. 1. - Long term evolutions for $>0.1 \mathrm{GeV}$ flux (upper panel) and the spin-down rate $\dot{f}$ (bottom panel). In the panels, the triangle (green), circle (blue) and square (red) symbols represent the results obtained by Al13, Zhao et al. (2017), and this work, respectively. The flux (upper panel) is calculated with 30 day time bin, and the spin-down rate (bottom panel) is obtained from the data with 60 day time bin for Al13 and with 70 day time bin for Zhao et al. (2017) and this work. The horizontal dashed-line in each panel and each stage denote the mean observed flux and spin-down rate. New results show that PSR J2021+4026 experienced a state change around MJD 58,150 (2018 February) again. 

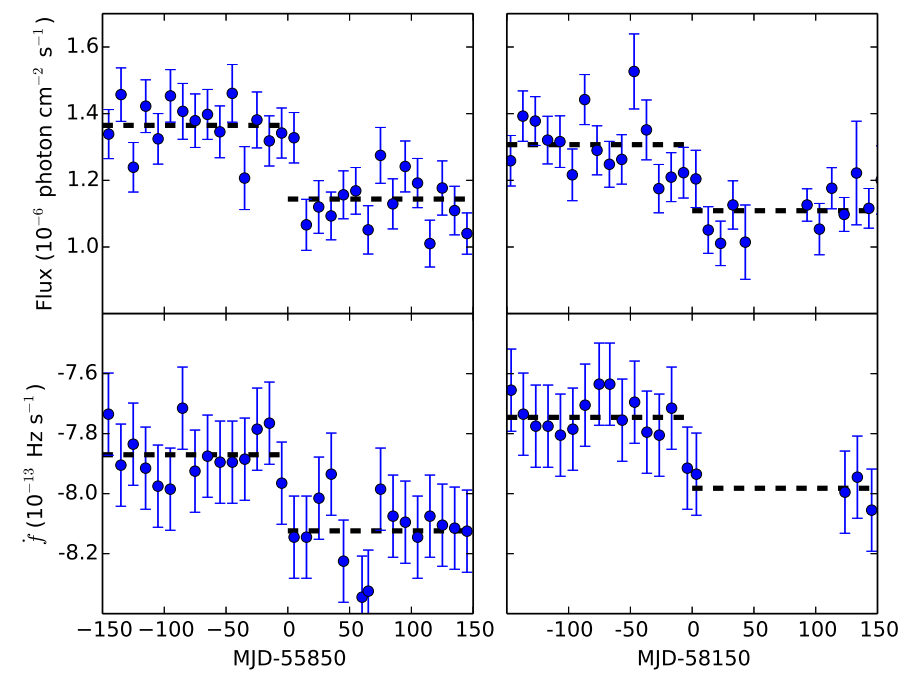

Fig. 2.- Evolutions for $>0.1 \mathrm{GeV}$ flux (upper panels) and the spin-down rate $\dot{f}$ (bottom panels) around the two state change. The flux is calculated with the data of 10 day time bin. In the bottom panel, the spin-down rate at each point is obtained with the data of 70 day time bin and the difference between the staring times of two neighbor points is 10 days, that is, two neighbor points share the data of 60 days. No Fermi-LAT data are available at around MJD 58,200. 


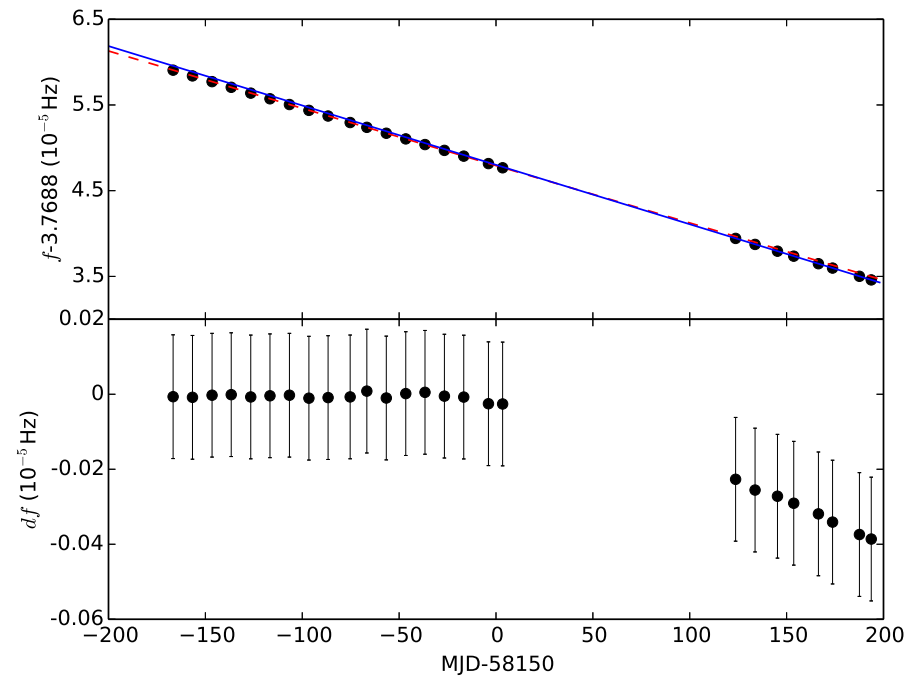

Fig. 3.- Spin frequency of PSR J2021+4026. Upper : The evolution of the spin frequency around 2018 state change. The dashed (red) and solid (blue) lines show the liner functions with a slope, $\dot{f}$, in the middle line (before state change) and in the last line (after state change) in Table 1. Bottom : Difference between the observed frequency and predicted frequency from the ephemeris (dashed line in the upper panel) before 2018 state change. The frequency is determined with the data of 70 day time bin, and two neighbor points overlap the 60 day data. The uncertainty is determined from the Fourier width, 1/70 days. No Fermi-LAT data are available around MJD 58,200. 
MJD 54,800-55,820 (HGF/LSD), 55,880-56,825 (LGF/HSD), 57,100-58,120 (HGF/LSD) and 58,270-58,540 (LGF/HSD), respectively. We find that the spin-down rate in the new HSD state after the 2018 state change is smaller than that of 2011 state change; the increase of the spin-down rate at 2018 state change, $\triangle \dot{f} / \dot{f}=3.1 \pm 1.1 \%$, is also smaller than the 2011 event $(5.6 \pm 0.9 \%)$.

Figure 2 is a close-up of the evolution of the gamma-ray flux with 10 day time bin size (top panels) and the spin-down rate (bottom panels) around two state change events. The spin-down rate at each point is calculated with the data of 70 day time bin and the starting time difference between two neighbor points is 10 days; namely, two neighbor points share the data of 60 days. For the previous event, we can see an abrupt change of the gamma-ray flux and the spin-down rate near MJD 55,850 with a timescale less than 10 days (Al13). For the 2018 event, the state change would have occurred at around MJD 58,150 with a timescale similar to the previous event, although abrupt change of the new event is less clear compared to the previous event.

The state change of PSR J2021+4026 could be triggered by the glitch, which is an abrupt change in the spin frequency of the neutron star. Figure 3 summarizes the evolution of the spin frequency around the 2018 state change. The dashed line and solid line in the upper

panel of the figure show the liner functions with the slope, $\dot{f}$, in the middle column (before state change) and in the last column (after state change), respectively, in Table 1 . The bottom panel shows the difference between the observed spin frequency and the predicted one from the ephemeris before the 2018 state change (dashed line in upper panel). We can see a change of the spin-down rate at around MJD 58,150. With the current uncertainty given by the data, however, we cannot confirm an apparent frequency jump around MJD 58,150. If there is a glitch event, its size, $\triangle f / f$, is much less than $10^{-7}$. Our result is consistent with the 2011 event (Al13).

\subsection{Pulse profile and phase-averaged spectrum}

The previous studies (Al13; Zhao et al. 2017) fit the gamma-ray pulse profile with two or three Gaussian components, and find that the pulse shape changed at the 2011 event. In particular, the intensity ratio of Peak 1 and Peak 2 was measured with $0.54 \pm 0.06$ for the $\mathrm{HGF} / \mathrm{LSD}$ state and with $0.24 \pm 0.03$ for the LGF/HSD state. The third Gaussian component was necessary in the fitting for the pulse profile of the HGF/LSD state before the 2011 event and it was not necessary for the LGF/HSD state. To examine the pulse profile after the state change in 2018, we generate the ephemeris between MJD 58,244 (2018, May 6st) and 58,722 (2019, August 27th) (Zhao et al. 2017 for the detailed method). Table 1 summarizes the 
Table 1: Ephemerides of PSR J2021+4026 derived from LAT data of MJD 55,857-56,943, 57,062-57,565 (Zhao et al. 2017) and 58,244-58,722 (this work). The numbers in parentheses denote $1 \sigma$ errors in the last digit.

\begin{tabular}{llll}
\hline \hline Parameter & & \\
\hline Right ascension, $\alpha \ldots \ldots \ldots \ldots \ldots$ & & $20: 21: 30.733$ & \\
Declination, $\delta \ldots \ldots \ldots \ldots \ldots \ldots$ & & $+40: 26: 46.04$ & \\
Valid MJD range $\ldots \ldots \ldots \ldots \ldots$ & $55,857-56,943$ & $57,062-57,565$ & $58,244-58,722$ \\
Pulse frequency, $f\left(\mathrm{~s}^{-1}\right) \ldots \ldots \ldots$ & $3.7689669240(2)$ & $3.7689112482(6)$ & $3.7688306913(3)$ \\
First derivative, $\dot{f}\left(10^{-13} \mathrm{~s}^{-2}\right) \ldots$ & $-8.1978(1)$ & $-7.738(1)$ & $-8.0224(4)$ \\
second derivative,,$\ddot{f}\left(10^{-22} \mathrm{~s}^{-3}\right) \ldots$ & $-0.19(2)$ & $3.5(3)$ & $-3.1(1)$ \\
Third derivative, $\dddot{f}\left(10^{-29} \mathrm{~s}^{-4}\right) \ldots$ & $0.572(7)$ & $-1.7(9)$ & $1.4(2)$ \\
Fourth derivative, $10^{-37} f^{(4)}\left(\mathrm{s}^{-4}\right)$ & $0.3(1)$ & $-6(9)$ & - \\
Epoch zero $(\mathrm{MJD}) \ldots \ldots \ldots \ldots \ldots$ & 56,400 & 57,200 & 58,400 \\
RMS timing residual $(\mu \mathrm{s}) \ldots \ldots$. & 2199.970 & 2795.366 & 1808.901 \\
Time system ............... & \multicolumn{3}{c}{ TDB (DE405) } \\
\hline
\end{tabular}

ephemerides at different epochs; the second and third columns present the ephemerides for MJD 55,875-56,943 (previous LGF/HSD state) and 57,062-57,565 (HGF/LSD state) reported in Zhao et al. (2017), and the fourth column presents the ephemeris obtained in the current study. As shown in section 3.1, the spin-down rate after 2018 state change is smaller than that measured after the 2011 event.

Figure 4 shows a pulse profile generated with $>0.1 \mathrm{GeV}$ energy bands after 2018 state change and its fit to the two Gaussian model (curved line) is overlaid. The fitting parameters in the different epochs are summarized in Table 2. We find that the peak ratio in the new state after the 2018 event is $P 1 / P 2 \sim 0.26 \pm 0.12$, which is consistent with the previous observation in the LGF/HSD state after 2011 state change. In the 2011 event, Peak 1 in the pulse profile changed the full width at half maximum (FWHM). In the 2018 event, we also observe the change of FWHM of Peak 1. The magnitude of the width change is consistent with the 2011 event within the error, as Table 2 shows.

Figure 5 shows the phase-averaged spectra before (MJD 57,800-58,100) and after (MJD $58,250-58,550)$ the 2018 state change, and Table 3 summarizes the fitting parameters of the different epochs using a power-law plus exponential cut-off function described by equation (11). The obvious change in the spectral properties are observed for the 2018 event as well as the 2011 event: the cut-off energy decreased and the photon index increased after the state 
Table 2: Parameters of Gaussian Fitting for Pulse Profiles of $E>0.1 \mathrm{GeV}$.

\begin{tabular}{ccccc}
\hline \hline & \multicolumn{2}{c}{2011} & \multicolumn{2}{c}{2018} \\
& HGF $/$ LSD $^{\mathrm{c}}$ & LGF $/ H S D^{\mathrm{d}}$ & ${\text { HGF } / \text { LSD }^{\mathrm{e}}}$ & LGF $/ H S D$ \\
\hline Peak 1 $^{\mathrm{a}}$ & $0.19 \pm 0.02$ & $0.13 \pm 0.02$ & $0.195 \pm 0.024$ & $0.119 \pm 0.027$ \\
Peak 2 $^{\mathrm{a}}$ & $0.176 \pm 0.007$ & $0.174 \pm 0.006$ & $0.152 \pm 0.010$ & $0.161 \pm 0.013$ \\
Peak 1/Peak 2 $^{\mathrm{b}}$ & $0.54 \pm 0.06$ & $0.24 \pm 0.03$ & $0.494 \pm 0.009$ & $0.260 \pm 0.118$ \\
\hline
\end{tabular}

a: FWHM. b: Ratio of amplitude. c: Al13, three Gaussian.

d: Al13, two Gaussian. e: Zhao et al. (2017), two Gaussian.

change. The similar change of the spectral properties was also observed in the 2011 event, and the observed flux level and the cut-off energy between the same states are consistent with each other. These results therefore suggest that gamma-ray emission of PSR J2021+4026 repeats the state change between two different states. If PSR J2021+4026 repeats the state change with the current observed time interval, the next recovery from the current LGF/HSD state to an HGF/LSD state may occur in 2021 and the next abrupt state change from the HGF/LSD state to a LGF/HSD state may happen in 2025.

\section{Discussion}

The timing and spectral analysis suggest that PSR J2021+4026 entered a new LGF/HSD state at around 2018 February 1st. Although the spin-down rate of current LGF/HSD state is slightly smaller than that after 2011 state change, the gamma-ray emission properties (spectrum and pulse profile) for the two LGF/HSD states are consistent to each other. Moreover, the properties of the emission properties and spin-down rate between previous two HGF/LSD states (i) before 2011 October and (ii) between 2014 December and 2018

Table 3: Parameters of spectral fitting. The parameter in 2011 event are taken from Zhao et al. (2017).

\begin{tabular}{ccc|cc}
\hline \hline & \multicolumn{2}{c|}{2011} & \multicolumn{2}{c}{2018} \\
Parameter & HGF/LSD & LGF/HSD & HGF/LSD & LGF/HSD \\
\hline Flux $\left(10^{-6} \mathrm{cts} \mathrm{cm}^{-2} \mathrm{~s}^{-1}\right)$ & $1.29 \pm 0.01$ & $1.15 \pm 0.01$ & $1.29 \pm 0.01$ & $1.12 \pm 0.01$ \\
Cutoff Energy $(\mathrm{MeV})$ & $2755 \pm 74$ & $2477 \pm 77$ & $2675 \pm 135$ & $2447 \pm 110$ \\
Photon index & $1.64 \pm 0.011$ & $1.66 \pm 0.014$ & $1.68 \pm 0.02$ & $1.75 \pm 0.02$ \\
\hline
\end{tabular}




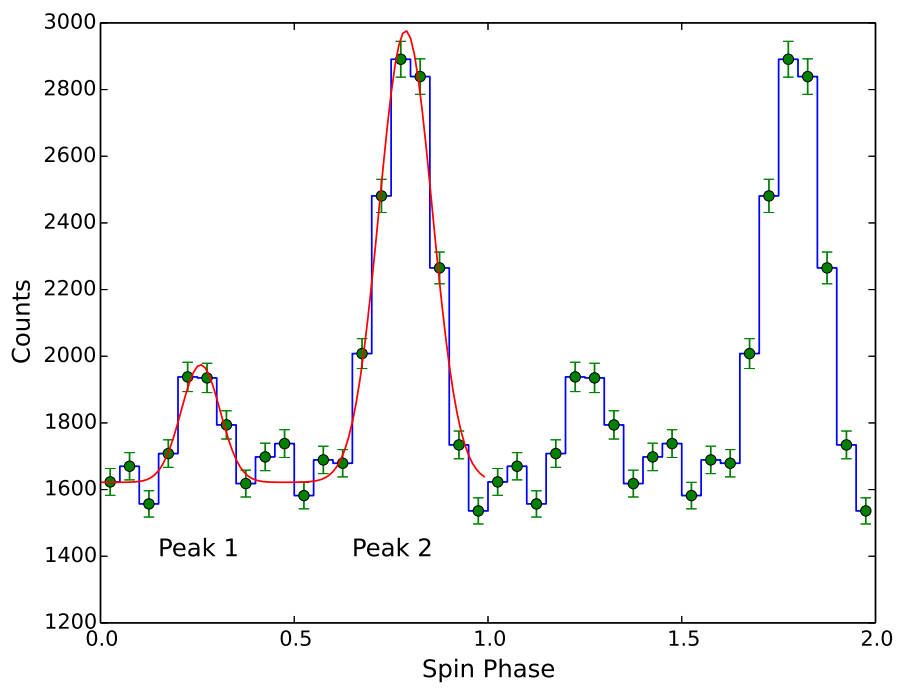

Fig. 4.- Pulse profile of J2021+4026 in MJD 58,244-58,722 (LGF/HSD state after 2018 state change). The pulse profile is generated with photon energy $>0.1 \mathrm{GeV}$. The curved line show the fitting results with two Gaussian components.

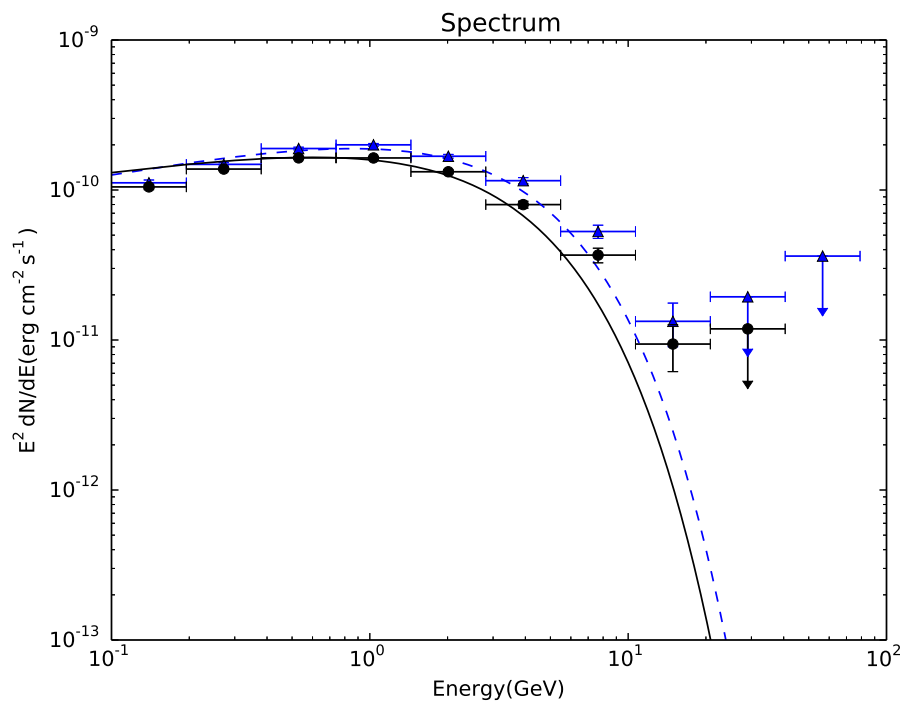

Fig. 5.- Phase averaged spectra around 2018 state change. Triangle and circle symbols present the spectra in HGF/LSD state (MJD 57,800-58,100) and LGF/HSD state (MJD 58, 250-58,550), respectively. The dashed line and solid line show the best fitting function for the HGF/LSD state and LFG/HSD state, respectively, Fitting parameters for each state are presented in Table 3. 
February are also consistent to each other. The long-term observation of Fermi-LAT suggests that (i) PSR J2021+4026 may repeat an abrupt state change from a HGF/LSD state to a LGF/HSD state with an time interval of $\tau_{\text {int }} \sim 6-7 \mathrm{yrs}$, (ii) the abrupt state change occurs over a timescale less than 10 days, and (iii) PSR J2021+4026 may stay at an LGF/HSD state for about three years and recover to an HGF/LSD over a timescale of a few months. Future continuum monitoring is required to obtain the solid conclusion of the time interval and timescale of the state change of PSR J2021+4026.

In this study, we cannot confirm the existence of the glitch at the state change. As we will discuss in this section, however, we expect that the state change is related to the neutron star's quake, which is probably related to the glitch of some pulsars. More than 180 glitching pulsars have been confirmed (Espinoza et al. 2011) 3 and about 50 Fermi-LAT pulsars show the glitch event (Kerr et al. 2015). However, the state changes of the gamma-ray emissions from other pulsars have not been confirmed yet. In the radio bands, on the other hand, it has been suggested that various observed phenomena, including emission mode change, nulling, and some timing noise, are related to the state change of the global magnetosphere. Lyne et al. (2010) report timing noise for 17 radio emitting pulsars, including the glitching pulsars, and they suggest that those timing behaviors are a result of switching (suddenly for some pulsars) between two different spin-down rates. They also demonstrate that the change of the spin-down rate of those "switching pulsars" is quasiperiodic with a timescale of days to years. Moreover, the timing noise of six pulsars is correlated to the change of the radio pulse profile, unlike the usual glitch in which no significant change of the pulse profile is observed. These results suggest that the structure of global magnetosphere of the state switching pulsars changes periodically with time. Although confirmation of the next state change from the current LGF/HSD state to HGF/LSD state is necessary, PSR J2021+4026 may also periodically switch between two different spin-down states.

It has been known that the change of the spin-down rate $|\triangle \dot{f}|$ of the glitching pulsars and switching pulsars (Lvne et al. 2010; Espinoza et al. 2011) is tightly correlated with the spin-down rate $|\dot{f}|$ (Figure 6). This would indicate that the change of the spin-down rate of switching pulsars is caused by a process similar to the glitch of some pulsars. We can see in Figure 6 that $|\triangle \dot{f}|$ of the switching pulsars tends to have larger value than the averaged values of the glitching pulsars, and the magnitude of $|\triangle \dot{f}|$ of PSR J2021+4026 is also larger than the values of the glitching pulsars with a similar spin-down rate. PSR J2021+4026 therefore may provide another opportunity to study the mechanisms of the state switching.

Besides the usual glitch process, several mechanisms have been proposed to change the

${ }^{3}$ http://www.jb.man.ac.uk/pulsar/glitches/gTable.html 
spin-down rate of the pulsars. For example, Ng et al. (2016) attribute the permanent-like increase in the spin-down rate of PSR J2021+4026 to the change of the inclination angle by $\triangle \alpha \sim 5^{\circ}$. The change of the spin-down rate can be also caused by the change of the global current structure. The spin-down power may be described as $L_{s d} \sim I V_{a}$, where $I$ is the current and $V_{a} \sim 2 \pi^{2} \mu / P_{s}^{2} c^{2}$, with $\mu$ being the magnetic dipole moment of the neutron star, is the available potential drop of the pulsar. The observed large change in the spin-down rate of PSR J2021+4026 may imply the change in the global current rather than the potential drop and the current in HSD sate increases by $\triangle I / I \sim \triangle \dot{f} / \dot{f} \sim 0.03$. In the pulsar magnetosphere, the magnitude of the current would be controlled by microphysical processes (e.g. pair-creation) of the particle acceleration regions, where the electric field along the magnetic field line exists. Several possible regions have been suggested for the acceleration/emission region, e.g. polar cap accelerator (Ruderman and Sutherland 1975; Daugherty and Harding 1996), slot gap (Harding et al. 2008; Harding and Kalapotharakos 2015), outer gap accelerator (Cheng et al. 1986; Takata et al. 2016) and current sheet region (Spitkovsky 2006). Since those different acceleration regions will be connected by the global current flow (Shibata 1995), a state change of one acceleration region will affect to other acceleration/emission regions and hence to the global magnetosphere structure. Since it has been thought that the gamma-ray emission occurs around the light cylinder, we may expect that some physical process around the light cylinder (e.g. magnetic reconnection at the Y-point, magnetosphere instability) triggers the state change of the pulsar. However, Fermi-LAT has already discovered > 200 gamma-ray emitting pulsars (The Fermi-LAT collaboration 2019), but only PSR J2021+4026 shows the switching behavior in both spin-down rate and emission properties (two Fermi-LAT pulsars, PSR J2043+2740 and J0742-2822, are probably switching pulsars, Lyne et al. 2010). Since PSR J2021+4026 has typical spin-down parameters in the gamma-ray pulsars, we should expect more variable gamma-ray pulsars, if the physical process around the light cylinder could trigger the state switching of PSR J2021+4026.

The state switching of PSR J2021+4026 is likely caused by change of the magnetic field structure of the polar cap region. Since the the polar cap region covers only about $0.05\left(P_{s} / 0.1 \mathrm{~s}\right)^{-1} \%$ of the stellar surface, the probability of the disturbance of the polar cap region by a glitch process (e.g. crust cracking) may be low. This could explain why switching pulsar, especially switching gamma-ray pulsar is rare. In Ruderman (1991a,b), the crust magnetic flux tube penetrates the type II super-conducting core. The neutron vortex lines in the core move as the pulsar spins down, and pull the magnetic flux tubes in the core with them. If the magnetic flux at the crust is immobile, the shear stress will be built up at the crust. The crust cracking will occur if the shear stress exceeds the crust elastic stress (Cheng \& Dai 1998),

$$
B_{c} \delta B / 8 \pi>\mu_{s} \theta_{\max } \frac{\ell}{R_{N S}}
$$


where $B_{c}$ is the average magnetic field strength in the quantized flux tubes at the core, $\delta B$ is the magnetic field strength creating the shear stress, $\mu_{s}$ is the shear modulus, $\theta_{\text {max }}$ is the strain before the cracking, $\ell$ is the size of the crust, and $R_{N S}$ is the radius of the neutron star. Hence, the magnetic field strength $\delta B$ just before the cracking is estimated to be

$$
\delta B \sim 3 \times 10^{11} \mathrm{G}\left(\frac{\mu_{s}}{10^{29} \mathrm{~g} \mathrm{~cm}^{-3}}\right)\left(\frac{\theta_{\max }}{10^{-3}}\right)\left(\frac{B_{c}}{10^{15} \mathrm{G}}\right)^{-1}\left(\frac{\ell}{10^{5} \mathrm{~cm}}\right) .
$$

After the cracking, the cracked plate will move to reduce the stress. The displacement $(\delta \ell)$ and the time interval $\left(\tau_{\text {int }}\right)$ between two successive abrupt state changes may be related with the stellar radius and characteristic age $\left(\tau_{s d}\right)$ of the pulsar by $\tau_{\text {int }} / \delta \ell \sim \tau_{s d} / R_{N S}$ (Ruderman 1991a,b). The displacement is rewritten as

$$
\delta \ell=10^{2} \mathrm{~cm}\left(\frac{\tau_{\text {int }}}{7 \mathrm{yrs}}\right)\left(\frac{\tau_{s d}}{70 \mathrm{kyrs}}\right)^{-1} .
$$

With the parameter of PSR J2021+4026, $\tau_{\text {int }} \sim 7 \mathrm{yrs}$ and $\tau_{s} \sim 70 \mathrm{kyrs}$, the displacement is estimated to be $\delta \ell \sim 100 \mathrm{~cm}$.

With the parameters of PSR J2021+4026, the radius of the polar cap is $R_{p c} \sim 2.8 \times$ $10^{4} \mathrm{~cm}$, and the size of the polar cap accelerator along the magnetic field line will be of the order of $\sim 10^{3} \mathrm{~cm}$ (Ruderman and Sutherland 1975). Hence the sudden displacement $\ell \sim 10^{2} \mathrm{~cm}$ of the cracked plate could affect to the polar cap structure. With a strong magnetic field at the polar cap region, the quantum electro dynamical processes such as the magnetic pair-creation process and/or the photon splitting process can be realized. The mean free path of such a magnetic process is sensitive to the photon energy, the strength of the magnetic field, and the angle between the propagation direction of the photon and direction of the magnetic field (Ruderman and Sutherland 1975; Timokhin and Harding 2019). If the crust cracking (or other process) would change the magnetic field structure or field strength in the polar cap region, the process affects to the created current and hence the spin-down rate. It is expected that the change of the current is very limited, that is, $\triangle I / I \ll 1$, since the magnitude of current $I$ will be controlled between the particle acceleration and the screening of the accelerating field by the pair-creation process and it will be always close to the Goldreich-Julian rate (Goldreich and Julian 1969).

The quasi-periodic change of the timing residual of the radio pulsars has been widely argued within the framework of the precession of the neutron star (Stairs et al. 2000; Link \& Epstein 2001; Jones 2012; Kerr et al. 2016). In the precession interpretation for a biaxial star, the precession period, $P_{p}$, is related to the spin period, $P_{s}$, by

$$
\frac{P_{s}}{P_{p}}=\frac{I_{0}}{I_{p}} \epsilon
$$


where $I_{0}$ and $I_{p}$ denote the characteristic moment of inertia, which neglects the small difference between different axes, of the star and of precessing body, respectively. The ellipticity $\epsilon$ is defined by $\epsilon=\triangle I_{p} / I_{0}$ with $\triangle I_{p}$ being the difference between the largest and smallest moments of inertia about the principal axes. With $P_{s}=0.265 \mathrm{~s}$ and $P_{p} \sim 6-7$ years that is observed time interval from an HGF/LSD state to a LGF/HSD state, the required ellipticity is of the order of $\epsilon \sim 10^{-9}$ if $I_{p} \sim I_{0}$, and it is similar to the values for other switching pulsars. The wobble angle $\theta$ is estimated to be of the order of $\theta \sim|\triangle \dot{f}| /|\dot{f}| \sim 2^{\circ}$, which is also similar to the required angle of well studied precession candidate, PSR B1828-11 (Link \& Epstein 2001).

We however argued that the change from an LSD state to an HSD state of PSR J2021+4026 happened over a timescale of $\sim 10$ days or less, which corresponds to the phase width $\delta \Phi \sim 10$ days/6.5years $\sim 0.004$. Such an abrupt change could be difficult to explain with a simple precession interpretation, since the variations in the spin-down rate given by the precession would be expressed with the fundamental and first harmonics of the Fourier components. Jones (2012) argues that an avalanche-like process such as the pair-creation process discussed above is probably enhanced at the some precession phase and magnifies the observed spin-down rate. Another possibility is that a glitch of PSR J2021+4026 excites new free precession and about three years of the LGF/HSD represents damping timescale of the precession. The damping timescale is estimated as $\tau_{d}=400-10^{4}$ precession period (Alpar \& Sauls 1988; Jones \& Andersson 2001). With $\tau_{d}=3$ years of PSR J2021+4026, the precession period is estimated to be $P_{p} \sim 0.1-2.6$ days. In this scenario, only a small part of the star will be precessed, $I_{p}<<I_{0}$, if the PSR J2021+4026 has a typical ellipticity $\epsilon \sim 10^{-8}-10^{-9}$. Unfortunately, it will be difficult to confirm the precession with $P_{p} \sim 1$ days for PSR J2021+4026 because of the radio quiet source.

The evolution of the spin-down rate is also discussed with the twisted pulsar magnetosphere, which are proposed for the evolutions of the X-ray luminosity and the spin-down rate of the magnetar after the X-ray outburst (Thompson et al. 2000; Beloborodov 2009). In this scenario, the neutron star quake would twist the closed magnetic field lines and the current flowing along the twisted magnetic field lines creates a toroidal current component. The induced toroidal current can provide an additional magnetic dipole moment, $\mu$. Since the spin-down rate is proportional to $\mu^{2}$, the twisted magnetosphere can increase the spin-down rate. This scenario can explain the evolution of the X-ray luminosity after the X-ray outburst of magnetar XTE J1810-197 (Beloborodov 2009) and the high-magnetic field radio pulsar PSR J1119-6127 (Wang et al. in preparation). For PSR J1119-6127, the pulsar underwent a large glitch at 2016 July X-ray outburst (Archibald et al. 2016), and the spin-down rate rapidly increased by a factor of $\sim 5-10$ in a timescale of a month. Then the pulsar recovered to the pre-burst rate over the following three months (Dai et al. 2018). In the twisted pulsar 
magnetosphere scenario, the timescale that the twisted magnetic field exists is characterized by the evolution timescale of ' $j$-bundle', and it for a canonical pulsar may be estimated as

$$
t_{t} \sim \frac{\phi_{0} \sin ^{2} \theta \mu}{c R_{N S} V_{e}} \sim \text { 7hours } \times \phi_{0}\left(\frac{\sin ^{2} \theta}{0.5}\right)\left(\frac{\mu}{5 \cdot 10^{30} \mathrm{G} \mathrm{cm}^{3}}\right)\left(\frac{e V_{e}}{10^{12} \mathrm{eV}}\right)^{-1}
$$

where $\phi_{0} \sim B_{t} / B$ is the twist angle with $B_{t}$ being the toroidal field, $\theta$ is the polar angle of the $j$-bundle, $R_{N S}$ is the neutron star radius and $V_{e}$ is the voltage to maintain the current flowing along the twisted magnetic field lines. For a canonical pulsar, the potential drop along the magnetic field line will develop so that the electrons/positrons emit the $\mathrm{GeV}$ photons via the curvature radiation process. The $\mathrm{GeV}$ photons will be converted into the electron/positron pairs by the magnetic pair-creation process and the pairs will stop the development of the potential drop along the magnetic field line. Near the stellar surface, the typical magnitude of the potential drop is of the order of $e V_{e} \sim 10^{12} \mathrm{eV}$. With the typical dipole moment, $\mu \sim 5 \times 10^{30} \mathrm{G} \mathrm{cm}^{3}$, of canonical pulsars, the timescale for the existence of the twisted magnetic field will be less than one day, which is difficult to explain the observed timescale $\sim 3$ years of high spin-down state of PSR J2021+4026. It is expected that if the abrupt state change of PSR J2021+4026 is caused by the twisting of the magnetosphere, the emission from the heated footprint contributes to the observed X-ray emission in a HSD state. In the previous studies of the X-ray emission from PSR J2021+4026 (Lin et al. 2013; Wang et al. 2018), there is no apparent difference between the X-ray emission properties in the HSD and LSD states. It is therefore that the twisted magnetosphere will not be the reason of the state change of PSR J2021+4026.

\section{Summary}

PSR J2021+4026 changed the state from a HGF/LSD state to a LGF/HSD state around MJD 58,150 (2018 February 1st), and the state change would occur over a timescale less than 10 days. The properties of the state change are similar to what observed in 2011 October, although the measured spin-down rate in the new state is slightly smaller than that measured after 2011 event. The long term evolution of the spin-down rate and gamma-ray emission properties suggest that PSR J2021+4026 repeats the state change every several years. The future monitoring is required to obtain the solid conclusion of time interval of the state change of PSR J2021+4026.

More than a dozen of switching pulsars have been discovered in the radio bands. PSR J2021+4026 will be the first switching pulsar discovered in the gamma-ray bands and provides unique opportunity to understand the mechanisms of the state switching. We argued that the shear stress building up at the crust will crack the plate that covers the polar 
cap region. The displacement of the cracked plate, $\delta \ell \sim 10^{2} \mathrm{~cm}$, will affect to the structure of the polar cap accelerator, whose size along the magnetic field line will be $\sim 10^{3} \mathrm{~cm}$. It would be also possible that the glitch of the neutron star excites new precession with a period of 0.1-2.6 days and the timescale of LGF/HSD state ( $\sim 3$ yrs) would present the damping timescale of the precession. If PSR J2021+4026 repeats the state change with the current observed time interval, the next recovery from the current LGF/HSD state to another HGF/LSD state may occur in 2021 and the next abrupt state change for a HGF/LSD state to a LGF/HSD state may happen in 2025.

We express our appreciation to an anonymous referee for useful comments and suggestions. J.T. and W.H.H. are supported by NSFC grants of the Chinese Government under 11573010, 11661161010, U1631103, and U1838102. A.K.H.K. is supported by the Ministry of Science and Technology of the Republic of China (Taiwan) through grants 1052119-M-007-028-MY3 and 106-2628-M-007-005. K.S.C. is supported by GRF grant under 17302315. C.Y.H. and K.L.L are supported by the National Research Foundation of Korea grant 2016R1A5A1013277. P.-H. T. T. is supported by NSFC through grants 11633007 and 11661161010 and U1731136.

\section{REFERENCES}

Abdo, A. A., Ackermann, M., Ajello, M., Atwood, W. B., Axelsson, M. et al., 2009a, ApJS, 183,46

Allafort, A., et al. 2013, ApJLetter, 777, L2

Alpar, M. A., \& Sauls, J. A. 1988, ApJ, 327, 723

Archibald, R. F., Kaspi, V. M., Tendulkar, S. P., \& Scholz, P. 2016, ApJ, 829, L21

Beloborodov, A. M. 2009, ApJ, 703, 1044

Cheng, K. S., Ho, C., \& Ruderman, M. 1986, ApJ, 300, 500

Cheng, K. S., \& Dai, Z. G. 1998, Physical Review Letters, 80, 18

de Jager, O. C., \& Büsching, I. 2010, A\&A, 517, L9

Dai, S., Johnston, S., Weltevrede, P., et al. 2018, MNRAS, 480, 3584

Daugherty, J. K. and Harding, A. K., 1996, ApJ, 458, 278

Espinoza, C. M., Lyne, A. G., Stappers, B. W. and Kramer, M., 2011, MNRAS, 414, 1689 
The Fermi-LAT collaboration 2019, arXiv:1902.10045

Goldreich, P. and Julian, W. H., 1969, ApJ, 157, 869

Harding, A.K.; Kalapotharakos, C., 2015, ApJ, 811,63

Harding, A. K.; Stern, J. V.; Dyks, J.; Frackowiak, M., 2008, ApJ, 680, 1378

Jones, D. I. 2012, MNRAS, 420, 2325

Jones, D. I., \& Andersson, N. 2001, MNRAS, 324, 811

Kerr, M., Hobbs, G., Johnston, S., \& Shannon, R. M. 2016, MNRAS, 455, 1845

Kerr, M., Ray, P. S., Johnston, S., et al. 2015, ApJ, 814, 128

Lin, L. C. C., Hui, C. Y., Hu, C. P., et al. 2013, ApJ, 770, L9

Link, B., \& Epstein, R. I. 2001, ApJ, 556, 392

Lyne, A., Hobbs, G., Kramer, M., Stairs, I. and Stappers, B., 2010, Science, 329, 408

Mattox, J. R., Bertsch, D. L., Chiang, J., et al. 1996, ApJ, 461, 396

Ng, C. W., Takata, J. and Cheng, K. S., ApJ, 2016, 825, 18

Ruderman, M. 1991a, ApJ, 366, 261

Ruderman, R. 1991b, ApJ, 382, 576

Ruderman, M. A. and Sutherland, P. G., 1975, ApJ, 196, 51

Stairs, I. H., Lyne, A. G., \& Shemar, S. L. 2000, Nature, 406, 484

Shibata, S. 1995, MNRAS, 276, 537

Spitkovsky, A., 2006, ApJLetter, 648, 51

Takata, J., Ng, C. W. and Cheng, K. S., 2016, MNRAS, 455, 4249

Thompson, C., Duncan, R. C., Woods, P. M., et al. 2000, ApJ, 543, 340

Timokhin, A. N., \& Harding, A. K. 2019, ApJ, 871, 12

Wang, H. H., Takata, J., Hu, C.-P., et al. 2018, ApJ, 856, 98

Zhao, J., Ng, C. W., Lin, L. C. C., et al. 2017, ApJ, 842, 53 
This preprint was prepared with the AAS LATEX macros v5.2. 


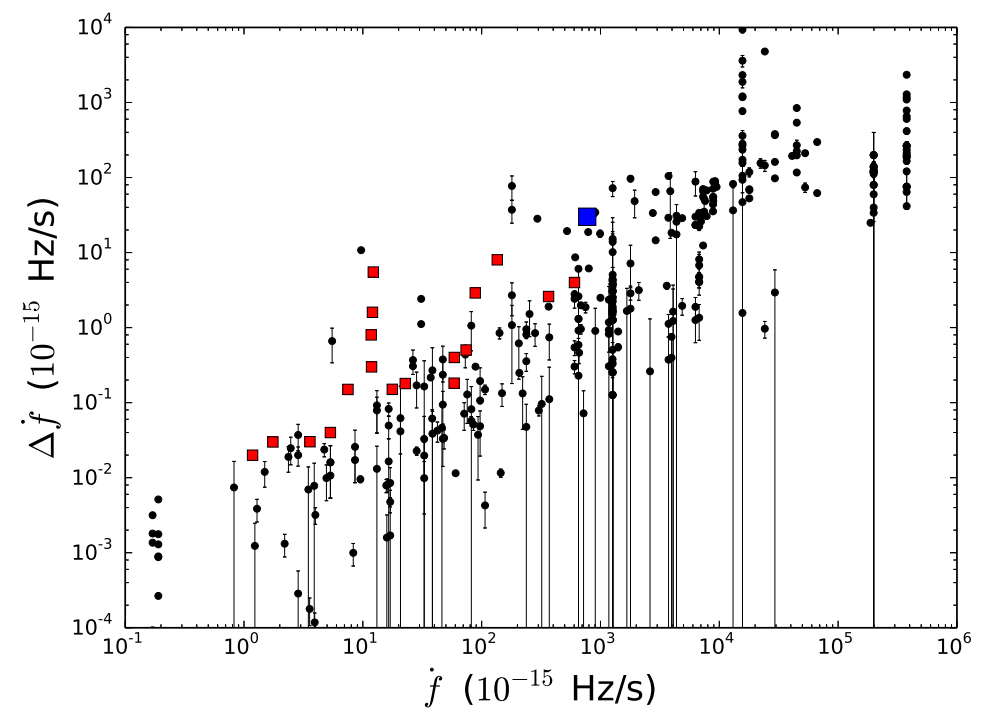

Fig. 6. - The change of the spin-down rate $(\triangle \dot{f})$ vs the spin-down rate $(\dot{f})$. Blue : PSR J2021+4026. Red: Switching pulsar listed in Lyne et al. (2010). Black: Glitching pulsars (Espinoza et al. 2011). 


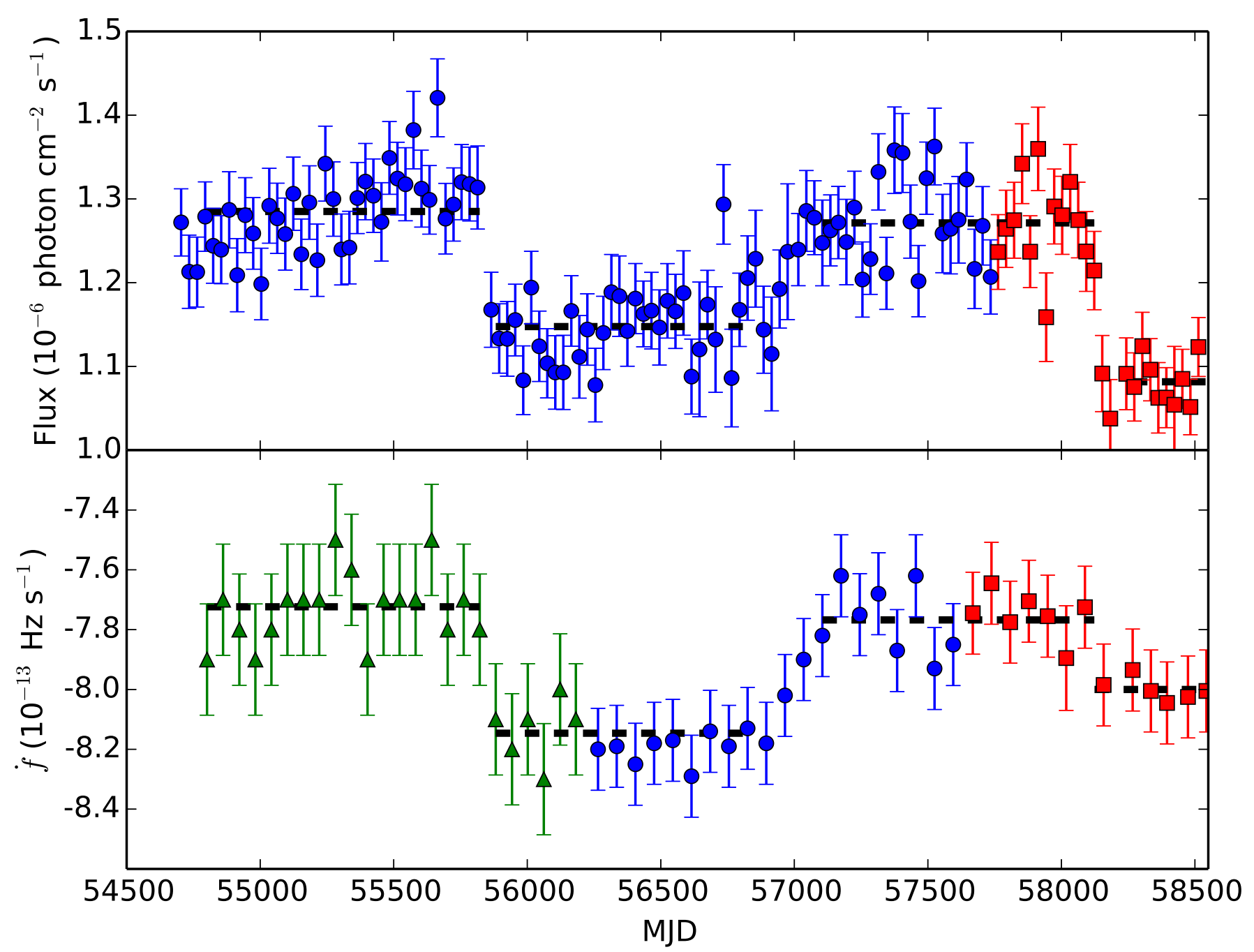

